\title{
On sum of monotone operator of type (FPV) and a maximal monotone operator
}

\author{
D. K. Pradhan and S. R. Pattanaik \\ Mathematics Department, National Institute of Technology, Rourkela, India
}

Received: 21 April 2016, Accepted: 14 august 2016

Published online: 12 November 2016.

\begin{abstract}
In the setting of a general real Banach space, we prove that the sum of a monotone operator $A$ of type (FPV) and a maximal monotone operator $B$ is maximal with $\operatorname{dom} A \cap \operatorname{int} \operatorname{dom} B \neq \phi$ and either $\operatorname{dom} B$ is open or for any $x \in \operatorname{dom} A \cap \operatorname{int} \operatorname{dom} B,\left\|x^{*}\right\| \leq|B(x)|$, $x^{*} \in A(x)$.
\end{abstract}

Keywords: Sum problem, Fitzpatrick function, maximal monotone operator, monotone operator of type (FPV).

\section{Introduction}

In monotone operator theory, the most studied and celebrated open problem concerns the maximal monotonicity of the sum of two maximal monotone operators. In 1970, Rockafellar proved it in reflexive space, i.e., the sum of two maximal monotone operators $A$ and $B$ with $\operatorname{dom} A \cap$ int $\operatorname{dom} B \neq \phi$ (Rockafellar's constraint qualification) is maximal monotone [10]. Therefore, it remains to study the sum theorem in nonreflexive spaces.

In [3], Borwein proves that the sum of two maximal monotone operators $A$ and $B$ is maximal monotone with int $\operatorname{dom} A \cap$ int $\operatorname{dom} B \neq \phi$. In [2], Bauschke, Wang and Yao prove that the sum of maximal monotone linear relation and the subdifferential operator of a sublinear function with Rockafellar's constraint qualification is maximal monotone. In [15], Yao extend the results in [2] to the subdifferential operator of any proper lower semicontinuous convex function. Yao [16] proves the that the sum of two maximal monotone operators $A$ and $B$ satisfying the conditions $A+N \overline{\operatorname{dom}} B$ is of type (FPV) and $\operatorname{dom} A \cap$ int $\operatorname{dom} B \neq \phi$ is maximal.

In [4], Borwein and Yao prove the maximal monotonicity of the sum of a maximal monotone linear relation and a maximal monotone with the assumptions that $\operatorname{dom} A \cap$ int $\operatorname{dom} B \neq \phi$. By relaxing the linearity from the result of [4], Borwein and Yao [6] prove the maximal monotonicity of $A+B$ provided that $A$ and $B$ are maximal monotone operators, $\operatorname{star}(\operatorname{dom} A) \cap \operatorname{int} \operatorname{dom} B \neq \phi$ and $A$ is of type (FPV). Also in [6] raises a question for further research on relaxing 'starshaped' hypothesis on $\operatorname{dom} A$.

In this paper we will prove that the sum of a monotone operator $A$ of type (FPV) and a maximal monotone operator $B$ is maximal with the assumption that $\operatorname{dom} B$ is open or for any $x \in \operatorname{dom} A \cap$ int $\operatorname{dom} B,\left\|x^{*}\right\| \leq|B(x)|$, where $x^{*} \in A(x)$. The remainder of this paper is organized as follows. In Section 2, we provide some auxiliary results and notions which will be used in our main results. In section 3 , main results are presented. 


\section{Basic notations and auxiliary results}

Suppose that $X$ is a real Banach space with norm, $\|$.$\| and \mathbb{U}_{\mathbb{X}}:=\{x \in X \mid\|x\|<1\}$ be the open unit ball in $X$. $X^{*}$ is the continuous dual of $X$ and $X$ and $X^{*}$ are paired by $\left\langle x, x^{*}\right\rangle=x^{*}(x)$ for $x \in X$ and $x^{*} \in X^{*}$. A sequence $x_{n}^{*} \in X^{*}$ is said to be weak $k^{*}$ convergence if there is some $x^{*} \in X^{*}$ such that $x_{n}^{*}(x) \rightarrow x^{*}(x)$ for all $x \in X$ and we denote it by $\neg w^{*}$. For a given subset $C$ of $X$ we denote interior of $C$ as int $C$, closure of $C$ as $\bar{C}$ and boundary of $C$ as bdry $C$. conv $C$, aff $C$ is the convex and affine hull of $C$. The intrinsic core or relative algebraic interior of $C$ is denoted by ${ }^{i} C$ [17] and is defined as ${ }^{i} C:=\{a \in C \mid \forall x \in \operatorname{aff}(C-C), \exists \delta>0, \forall \lambda \in[0, \delta]: a+\lambda x \in C\}$. And

$$
{ }^{i c} C:=\left\{\begin{array}{ll}
{ }^{i} C, & \text { if aff } \mathrm{C} \text { is closed, } \\
\phi, & \text { otherwise }
\end{array} .\right.
$$

For $0 \in \operatorname{Core} C$ iff $\bigcup_{\lambda>0} \lambda C=X$. Also we denote the distance function by $\operatorname{dist}(x, C):=\inf _{c \in C}\|x-c\|$ and $|C|=\inf _{c \in C}\|c\|$. For any $C, D \subseteq X, C-D=\{x-y \mid x \in C, y \in D\}$. Let $A: X \rightrightarrows X^{*}$ be a set-valued operator (also known as multifunction or point-to-set mapping) from $X$ to $X^{*}$, i.e., for every $x \in X, A x \subseteq X^{*}$. Domain of $A$ is denoted as $\operatorname{dom} A:=\{x \in X \mid A x \neq \phi\}$ and range of $A$ is $\operatorname{ran} A=\left\{x^{*} \in A x \mid x \in \operatorname{dom} A\right\}$. Graph of $A$ is denoted as gra $A=\left\{\left(x, x^{*}\right) \in X \times X^{*} \mid x^{*} \in A x\right\}$. A is said to be linear relation if gra $A$ is a linear subspace. The set-valued mapping $A: X \rightrightarrows X^{*}$ is said to be monotone if

$$
\left\langle x-y, x^{*}-y^{*}\right\rangle \geq 0, \quad \forall\left(x, x^{*}\right),\left(y, y^{*}\right) \in \operatorname{gra} A .
$$

Let $A: X \rightrightarrows X^{*}$ be monotone and $\left(x, x^{*}\right) \in X \times X^{*}$ we say that $\left(x, x^{*}\right)$ is monotonically related to gra $A$ if

$$
\left\langle x-y, x^{*}-y^{*}\right\rangle \geq 0, \quad \forall\left(y, y^{*}\right) \in \operatorname{gra} A
$$

And a set valued mapping $A$ is said to maximal monotone if $A$ is monotone and $A$ has no proper monotone extension(in the sense of graph inclusion). In other words $A$ is maximal monotone if for any $\left(x, x^{*}\right) \in X \times X^{*}$ is monotonically related to $\operatorname{gra} A$ then $\left(x, x^{*}\right) \in \operatorname{gra} A$. We say that $A$ is of type (FPV) if for every open set $U \subseteq X$ such that $U \cap \operatorname{dom} A \neq \phi$, $x \in U$ and $\left(x, x^{*}\right)$ is monotonically related to $\operatorname{gra} A \cap U \times X^{*}$, then $\left(x, x^{*}\right) \in \operatorname{gra} A$. Every monotone operators of type (FPV) are maximal monotone operators [13].

Let $f: X \rightarrow]-\infty,+\infty]$ be a function and its domain is defined as $\operatorname{dom} f:=f^{-1}(\mathbb{R}) . f$ is said to be proper if $\operatorname{dom} f \neq \phi$. Let $f$ be any proper convex function then the subdifferential operator of $f$ is defined as $\partial f: X \rightrightarrows X^{*}: x \mapsto\left\{x^{*} \in X^{*} \mid\left\langle y-x, x^{*}\right\rangle+f(x) \leq f(y), \forall y \in X\right\}$. Subdifferential operators are of type (FPV)[13]. For every $x \in X$, the normal cone operator at $\mathrm{x}$ is defined by $N_{C}(x)=\left\{x^{*} \in X^{*} \mid \sup _{c \in C}\left\langle c-x, x^{*}\right\rangle \leq 0\right\}$, if $x \in C$; and $N_{C}(x)=\phi$, if $x \notin C$. Also it may be verified that the normal cone operator is of type (FPV) [13]. For $x, y \in X$, we denote $[x, y]:=\{t x+(1-t) y \mid 0 \leq t \leq 1\}$ and star or center of $C$ as $\operatorname{star} C:=\{x \in C \mid[x, c] \subseteq C, \forall c \in C\}[17]$.

We denote the projection map by $P_{X}: X \times X^{*} \rightarrow X$ by $P_{X}\left(x, x^{*}\right)=x$. For any two $A$ and $B$ monotone operators, the sum operator is defined as $A+B: X \rightrightarrows X^{*}: x \mapsto A x+B x=\left\{a^{*}+b^{*} \mid a^{*} \in A x\right.$ and $\left.b^{*} \in B x\right\}$. It may be checked that $A+B$ is monotone.

Fact 1. [8, Theorem 2.28] Let $A: X \rightrightarrows X^{*}$ be monotone with int $\operatorname{dom} A \neq \phi$. Then $\mathrm{A}$ is locally bounded at $x \in \operatorname{int} \operatorname{dom} A$, i.e., there exist $\delta>0$ and $K>0$ such that

$$
\sup _{y^{*} \in A y}\left\|y^{*}\right\| \leq K, \quad \forall y \in\left(x+\delta \mathbb{U}_{\mathbb{X}}\right) \cap \operatorname{dom} A .
$$


Fact 2. [Fitzpatrick] [7, Corollary 3.9] Let $A: X \rightrightarrows X^{*}$ be maximal monotone, and $F_{A}: X \times X^{*} \rightarrow(-\infty,+\infty]$ defined by

$$
F_{A}\left(x, x^{*}\right)=\sup _{\left(a, a^{*}\right) \in g r a A}\left(\left\langle x, a^{*}\right\rangle+\left\langle a, x^{*}\right\rangle-\left\langle a, a^{*}\right\rangle\right)
$$

which is the Fitzpatrick function associated with A. Then for every $\left(x, x^{*}\right) \in X \times X^{*}$, the inequality $\left\langle x, x^{*}\right\rangle \leq F_{A}\left(x, x^{*}\right)$ is true, and equality holds if and only if $\left(x, x^{*}\right) \in \operatorname{gra} A$.

Fact 3. [14, Theorem 3.4 and Corollary 5.6], or [13, Theorem 24.1(b)] Let $A, B: X \rightrightarrows X^{*}$ be maximal monotone operator. Assume $\bigcup_{\lambda>0} \lambda\left[P_{X}\left(\operatorname{dom} F_{A}\right)-P_{X}\left(\operatorname{dom} F_{B}\right)\right]$ is a closed subspace. If $F_{A+B} \geq\langle.,$.$\rangle on X \times X^{*}$, then $A+B$ is maximal monotone.

Fact 4. [17, Theorem 1.1.2(ii)] Let $C$ be a convex subset of $X$. If $a \in \operatorname{int} C$ and $x \in \bar{C}$, then $[a, x[\subset \operatorname{int} C$.

Fact 5. [Rockafellar][9, Theorem 1] or [13, Theorem 27.1 and Theorem 27.3] Let $A: X \rightrightarrows X^{*}$ be maximal monotone with int $\operatorname{dom} A \neq \phi$. Then int $\operatorname{dom} A=\operatorname{int} \overline{\operatorname{dom} A}$; and int $\operatorname{dom} A$ and $\overline{\operatorname{dom} A}$ is convex.

Fact 6. [6, Proposition 3.1] Let $A: X \rightrightarrows X^{*}$ be of type (FPV), and let $B: X \rightrightarrows X^{*}$ be maximally monotone. Suppose that $\operatorname{dom} A \cap \operatorname{int} \operatorname{dom} B \neq \phi$. Let $\left(z, z^{*}\right) \in X \times X^{*}$ with $z \in \overline{\operatorname{dom} B}$. Then $F_{A+B}\left(z, z^{*}\right) \geq\left\langle z, z^{*}\right\rangle$.

Fact 7. [1, Lemma 2.5] Let $C$ be a nonempty closed convex subset of $X$ such that int $C \neq \phi$. Let $c_{0} \in \operatorname{int} C$ and suppose that $z \in X \backslash C$. Then there exists $\lambda \in] 0,1\left[\right.$ such that $\lambda c_{0}+(1-\lambda) z \in$ bdry $C$.

Fact 8. [13, Theorem 44.2] Let $A: X \rightrightarrows X^{*}$ be of type (FPV). Then

$$
\overline{\operatorname{dom} A}=\overline{\operatorname{conv}(\operatorname{dom} A)}=\overline{P_{X}\left(\operatorname{dom} F_{A}\right)}
$$

Fact 9. [6, Lemma 2.10] Let $A: X \rightrightarrows X^{*}$ be monotone, and Let $B: X \rightrightarrows X^{*}$ be maximally monotone. Let $\left(z, z^{*}\right) \in X \times X^{*}$. Suppose $x_{0} \in \operatorname{dom} A \cap \operatorname{int} \operatorname{dom} B$ and that there exists a sequence $\left(a_{n}, a_{n}^{*}\right)_{n \in \mathbb{N}}$ in $\operatorname{gra} A \cap\left(\operatorname{dom} B \times X^{*}\right)$ such that $\left(a_{n}\right)_{n \in \mathbb{N}}$ converges to a point in $\left[x_{0}, z\left[\right.\right.$, while $\left\langle z-a_{n}, a_{n}^{*}\right\rangle \longrightarrow \infty$. Then $F_{A+B}\left(z, z^{*}\right)=+\infty$.

Fact 10. [6, Lemma 2.12] Let $A: X \rightrightarrows X^{*}$ be of type (FPV). Suppose $x_{0} \in \operatorname{dom} A$ but that $z \notin \overline{\operatorname{dom} A}$. Then there exists a sequence $\left(a_{n}, a_{n}^{*}\right)_{n \in \mathbb{N}}$ in gra $A$ so that $\left(a_{n}\right)_{n \in \mathbb{N}}$ converges to a point in $\left[x_{0}, z\left[\right.\right.$ and $\left\langle z-a_{n}, a_{n}^{*}\right\rangle \longrightarrow+\infty$.

Fact 11. [The Banach-Alaoglu Theoerem][11, Theorem 3.15] The closed unit ball in $X^{*}, B_{X}^{*}$ is weak star compact.

Fact 12. [16] Let $A: X \rightrightarrows X^{*}$ be maximally monotone and $z \in \overline{\operatorname{dom} A} \backslash \operatorname{dom} A$. Then for every sequence $\left(z_{n}\right)_{n \in \mathbb{N}}$ in $\operatorname{dom} A$ such that $z_{n} \rightarrow z$, we have $\lim _{n \rightarrow \infty} \inf \left\|A\left(z_{n}\right)\right\|=+\infty$.

Proof. Suppose to the contrary that there exists a sequence $z_{n_{k}}^{*} \in A\left(z_{n_{k}}\right)$ and $L>0$ such that $\sup _{k \in \mathbb{N}}\left\|z_{n_{k}}^{*}\right\| \leq L$. By Fact 2, there exists a weak* convergent subnet, $\left(z_{\beta}^{*}\right)_{\beta \in J}$ of $z_{n_{k}}^{*}$ such that $z_{\beta}^{*} \neg_{w}^{*} z_{\infty}^{*} \in X^{*}$. By [5, Fact 3.5], we have $\left(z, z_{\infty}^{*}\right) \in \operatorname{gra} A$, which is a contradiction to our assumption that $z \notin \operatorname{dom} A$.

Fact 13. [6, Lemma 2.11] Let $A: X \rightrightarrows X^{*}$ be of type (FPV), and Let $B: X \rightrightarrows X^{*}$ be maximally monotone. Let $\left(z, z^{*}\right) \in$ $X \times X^{*}$. Suppose $x_{0} \in \operatorname{dom} A \cap$ int $\operatorname{dom} B$. Assume that there exists a sequence $\left(a_{n}\right)_{n \in \mathbb{N}}$ in $\operatorname{dom} A \cap \operatorname{dom} B$ and $\beta \in[0,1]$ such that $a_{n} \rightarrow \beta z+(1-\beta) x_{0}$ and $a_{n} \in$ bdry $\operatorname{dom} B$ Then $F_{A+B}\left(z, z^{*}\right)=+\infty$. 
Fact 14. [16, Proposition 3.1] Let $A: X \rightrightarrows X^{*}$ be of type (FPV), and Let $B: X \rightrightarrows X^{*}$ be maximally monotone. Let $\left(z, z^{*}\right) \in X \times X^{*}$. Suppose $x_{0} \in \operatorname{dom} A \cap$ int $\operatorname{dom} B$. Assume that there exists a sequence $\left(a_{n}\right)_{n \in \mathbb{N}}$ in $\operatorname{dom} A \cap[\overline{\operatorname{dom} B} \backslash \operatorname{dom} B]$ and $\beta \in[0,1]$ such that $a_{n} \rightarrow \beta z+(1-\beta) x_{0}$ Then $F_{A+B}\left(z, z^{*}\right) \geq\left\langle z, z^{*}\right\rangle$.

\section{Our main results}

We first prove the useful results which play an important role to prove our main results.

Lemma 1. Let $A$ be any subset of $X, 0 \in \operatorname{int} \bar{A}=\operatorname{int} A$ and $\bar{A}$ is convex. Then $x \in \bar{A}$ if and only if $x \in \operatorname{int}(1+\varepsilon) A$ for every $0<\varepsilon<1$.

Proof. First we show that $A \subset(1+\varepsilon) A$, for every $0<\varepsilon<1$. Let $z \in A$ and assume on the contrary that there exist $0<\varepsilon<1$ such that $z \notin(1+\varepsilon) A$ i.e., $\frac{z}{(1+\varepsilon)} \notin A$. By Fact 2 and hypothesis, $t z \in \operatorname{int} \bar{A}=\operatorname{int} A, \quad \forall 0 \leq t<1$. In particular, for $t=\frac{1}{1+\varepsilon}<1$ we have $t z \notin A$ which is a contradiction. Now we show that $\bar{A} \subset \operatorname{int}(1+\varepsilon) A$. Let $x \in \bar{A}$. If $x \in \operatorname{int} A$, then clearly $x \in \operatorname{int}(1+\varepsilon) A$. If $x \in \operatorname{bdry} A$, then we show $x \in \operatorname{int}(1+\varepsilon) A$. On the contrary, if $x \in \operatorname{bdry}(1+\varepsilon) A$ i.e., $\frac{x}{1+\varepsilon} \in \operatorname{bdry} A$ for some $\varepsilon$. By $0 \in \operatorname{int} A$ and $x \in \bar{A}$ and Fact $2, t x \in \operatorname{int} A, \forall 0 \leq t<1$. For $t=\frac{1}{1+\varepsilon}, t x \in \operatorname{bdry} A$ which is a contradiction. Hence, $x \in \operatorname{int}(1+\varepsilon) A$ for every $0<\varepsilon<1$. Conversely, $x \in \operatorname{int}(1+\varepsilon) A$, for every $0<\varepsilon<1$. For $\varepsilon=\frac{1}{n}, n=1,2,3, \ldots$ $x \in \operatorname{int}\left(1+\frac{1}{n}\right) A=\operatorname{int} A_{n}$. Thence, there exist $U\left(x, r_{n}\right) \subset A_{n}$. Choose $y_{n} \in A_{n}$ such that $y_{n} \in U\left(x, r_{n}\right)$ and $r_{n}$ such that $r_{n} \rightarrow 0$ as $n \rightarrow \infty$. Since $y_{n} \in A_{n}$ then there exists $x_{n} \in A$ such that $y_{n}=\left(1+\frac{1}{n}\right) x_{n}$ which implies $x_{n}=\frac{y_{n}}{1+\frac{1}{n}} \rightarrow x$. Hence $x \in \bar{A}$.

The proof of the following Lemma 2 closely follows the lines of the proof of [6, Proposition 3.2].

Lemma 2. Let $A: X \rightrightarrows X^{*}$ be of type (FPV), and let $B: X \rightrightarrows X^{*}$ be maximally monotone. Let $\left(z, z^{*}\right) \in X \times X^{*}, x_{0} \in$ $\operatorname{dom} A \cap \operatorname{dom} B$ and $\operatorname{dom} B$ is open. Assume that there exists $\left(a_{n}\right)_{n \in \mathbb{N}} \in \overline{\operatorname{dom} A} \cap$ bdry $\overline{\operatorname{dom} B}$ such that it converges to a point in $\left[x_{0}, z\right]$. Then $F_{A+B}\left(z, z^{*}\right) \geq\left\langle z, z^{*}\right\rangle$.

Proof. Assume to the contrary

$$
F_{A+B}\left(z, z^{*}\right)<\left\langle z, z^{*}\right\rangle
$$

By the necessary translation if necessary, we can suppose that $x_{0}=0 \in \operatorname{dom} A \cap$ int $\operatorname{dom} B$ and $(0,0) \in \operatorname{gra} A \cap \operatorname{gra} B$. By the assumption that, there exists $0 \leq \beta<1$ such that

$$
a_{n} \longrightarrow \beta z
$$

Since $0 \in \operatorname{int} \operatorname{dom} B$ and by (1) Fact 2, we have

$$
0<\beta<1 \text { and } \beta z \neq 0
$$

Since $a_{n} \in \operatorname{dom} A$ we set

$$
y_{0}:=\beta z \text { and }
$$

By $0 \in$ int $\operatorname{dom} B$ and (3), there exists $0<\rho_{0} \leq\left\|y_{0}\right\|$ such that

$$
\rho_{0} \mathbb{U}_{\mathbb{X}} \subseteq \operatorname{dom} B
$$

Now we show that there exists $\beta \leq \delta_{n} \in\left[1-\frac{1}{n}, 1[\right.$ such that

$$
H_{n} \subseteq \operatorname{dom} B
$$

where

$$
H_{n}:=\delta_{n} \beta z+\left(1-\delta_{n}\right) \rho_{0} \mathbb{U}_{\mathbb{X}} .
$$


By Fact 2 and Fact 2, we have for every $s \in(0,1)$,

$$
s \beta z+(1-s) \rho_{0} \mathbb{U}_{\mathbb{X}} \subseteq \text { int } \overline{\operatorname{dom} B}=\text { int } \operatorname{dom} B .
$$

Hence (6) holds.

Since $a_{n} \rightarrow y_{0}$ and $\delta_{n} \beta z=v_{n}$ (say) by (7), $v_{n} \rightarrow y_{0}$. Then we can suppose that

$$
\left\|v_{n}\right\| \leq\left\|y_{0}\right\|+1 \leq\|z\|+1, \quad \forall n \in \mathbb{N}(b y(4)) .
$$

Next we show that there exists $\left(\widetilde{a_{n}},{\widetilde{a_{n}}}^{*}\right)_{n \in \mathbb{N}}$ in gra $A \cap\left(H_{n} \times X^{*}\right)$ such that

$$
\left\langle z-{\widetilde{a_{n}}},{\widetilde{a_{n}}}^{*}\right\rangle \geq-K_{0}\left\|a_{n}^{*}\right\|
$$

where $K_{0}=\frac{1}{\beta^{2}}(2\|z\|+2)$. Since $\delta_{n} \beta z=v_{n} \in H_{n}$ and $a_{n}^{*} \in X^{*}$, then we consider two cases.

Case 1. $\left(v_{n}, a_{n}^{*}\right) \in \operatorname{gra} A$. Take $\left(\widetilde{a_{n}},{\widetilde{a_{n}}}^{*}\right):=\left(v_{n}, a_{n}^{*}\right)$.

$$
\begin{aligned}
\left\langle z-\widetilde{a_{n}},{\widetilde{a_{n}}}^{*}\right\rangle & =\left\langle z-v_{n}, a_{n}^{*}\right\rangle \\
& \geq-\left\|z-v_{n}\right\|\left\|a_{n}^{*}\right\| \\
& \geq-(2\|z\|+2)\left\|a_{n}^{*}\right\| \text { by equation(8). } \\
& \geq-K_{0}\left\|a_{n}^{*}\right\| .
\end{aligned}
$$

Hence (9) holds.

Case 2. $\left(v_{n}, a_{n}^{*}\right) \notin \operatorname{gra} A$. By Fact 2 and by the assumption $a_{n} \in \overline{\operatorname{dom} A}$, we get $v_{n}=\delta_{n} \beta z \in \overline{\operatorname{dom} A}$. Therefore, $H_{n} \cap \operatorname{dom} A \neq \phi$. Since $\left(v_{n}, a_{n}^{*}\right) \notin \operatorname{gra} A$ and $v_{n} \in H_{n}$, by using $(F P V)$ property there exists $\left(\widetilde{a_{n}},{\widetilde{a_{n}}}^{*}\right) \in \operatorname{gra} A \cap\left(H_{n} \times X^{*}\right)$ such that

$$
\left\langle v_{n}-{\widetilde{a_{n}}} a_{n}^{*}-{\widetilde{a_{n}}}^{*}\right\rangle<0
$$

Thus, we have

$$
\begin{aligned}
\left\langle v_{n}-\widetilde{a_{n}},{\widetilde{a_{n}}}^{*}-a_{n}^{*}\right\rangle>0 & \Rightarrow\left\langle v_{n}-\widetilde{a_{n}}, \widetilde{a_{n}}{ }^{*}\right\rangle>\left\langle v_{n}-\widetilde{a_{n}}, a_{n}^{*}\right\rangle \\
& \Rightarrow\left\langle\delta_{n} \beta z-\delta_{n} \beta \widetilde{a_{n}}+\delta_{n} \beta \widetilde{a_{n}}-\widetilde{a_{n}},{\widetilde{a_{n}}}^{*}\right\rangle>\left\langle v_{n}-\widetilde{a_{n}}, a_{n}^{*}\right\rangle \\
& \Rightarrow\left\langle\delta_{n} \beta\left(z-\widetilde{a_{n}}\right)-\left(1-\delta_{n} \beta\right) \widetilde{a_{n}}, \widetilde{a_{n}}{ }^{*}\right\rangle>\left\langle v_{n}-\widetilde{a_{n}}, a_{n}^{*}\right\rangle \\
& \Rightarrow\left\langle\delta_{n} \beta\left(z-\widetilde{a_{n}}\right), \widetilde{a_{n}}{ }^{*}\right\rangle>\left(1-\delta_{n} \beta\right)\left\langle\widetilde{a_{n}}, \widetilde{a_{n}}{ }^{*}\right\rangle+\left\langle v_{n}-\widetilde{a_{n}}, a_{n}^{*}\right\rangle .
\end{aligned}
$$

Since $\beta \leq \delta_{n}<1,(0,0) \in \operatorname{gra} A$ and $\left(\widetilde{a_{n}},{\widetilde{a_{n}}}^{*}\right) \in \operatorname{gra} A$, applying monotonicity of $A$, we have

$$
\begin{aligned}
\left\langle\delta_{n} \beta\left(z-\widetilde{a_{n}}\right),{\widetilde{a_{n}}}^{*}\right\rangle \geq\left\langle v_{n}-\widetilde{a_{n}}, a_{n}^{*}\right\rangle & \Rightarrow\left\langle z-\widetilde{a_{n}},{\widetilde{a_{n}}}^{*}\right\rangle \geq \frac{1}{\delta_{n} \beta}\left\langle v_{n}-\widetilde{a_{n}}, a_{n}^{*}\right\rangle . \\
& \Rightarrow\left\langle z-\widetilde{a_{n}},{\widetilde{a_{n}}}^{*}\right\rangle \geq-\frac{1}{\delta_{n} \beta}\left\|v_{n}-\widetilde{a_{n}}\right\|\left\|a_{n}^{*}\right\| \\
& \Rightarrow\left\langle z-\widetilde{a_{n}},{\widetilde{a_{n}}}^{*}\right\rangle \geq-\frac{1}{\beta^{2}}\left\|v_{n}-\widetilde{a_{n}}\right\|\left\|a_{n}^{*}\right\| .
\end{aligned}
$$


Since $v_{n}, \widetilde{a_{n}} \in H_{n}$, then we have $\widetilde{a_{n}} \rightarrow y_{0}$ and we can suppose that

$$
\left\|\widetilde{a_{n}}\right\| \leq\left\|y_{0}\right\|+1 \leq\|z\|+1, \forall n \in \mathbb{N}
$$

Appealing to equation (11), we have

$$
\begin{aligned}
\left\langle z-\widetilde{a_{n}},{\widetilde{a_{n}}}^{*}\right\rangle & \geq-\frac{1}{\beta^{2}}(2\|z\|+2)\left\|a_{n}^{*}\right\| \\
& =-K_{0}\left\|a_{n}^{*}\right\| .
\end{aligned}
$$

Since $\beta z \in$ bdry $\operatorname{dom} B$ and by hypothesis, we have $\beta z \in \overline{\operatorname{dom} B} \backslash \operatorname{dom} B$. Then by Fact 2 we have,

$$
\inf \left\|B\left(H_{n}\right)\right\| \geq K_{0}\left\|a_{n}^{*}\right\| n
$$

Since $\widetilde{a_{n}} \in H_{n}$, equation (6) implies that $\widetilde{a_{n}} \in$ int $\operatorname{dom} B$ and $\widetilde{a_{n}} \in \operatorname{dom} A$. Again since $\widetilde{a_{n}} \in H_{n}$ then take $b_{n}^{*} \in B\left(\widetilde{a_{n}}\right)$ by (13),

$$
\left\|b_{n}^{*}\right\| \geq n K_{0}\left\|a_{n}^{*}\right\|
$$

We compute

$$
\begin{aligned}
F_{A+B}\left(z, z^{*}\right) & =\sup _{\left\{\widetilde{a_{n}}{ }^{*}+b_{n}^{*} \in(A+B)\left(\widetilde{a_{n}}\right)\right\}}\left[\left\langle\widetilde{a_{n}}, z^{*}\right\rangle+\left\langle z-\widetilde{a_{n}},{\widetilde{a_{n}}}^{*}\right\rangle+\left\langle z-\widetilde{a_{n}}, b_{n}^{*}\right\rangle\right] \\
& \geq\left[\left\langle\widetilde{a_{n}}, z^{*}\right\rangle+\left\langle z-\widetilde{a_{n}},{\widetilde{a_{n}}}^{*}\right\rangle+\left\langle z-\widetilde{a_{n}}, b_{n}^{*}\right\rangle\right] .
\end{aligned}
$$

By (9) and (14), we have

$$
\begin{aligned}
& F_{A+B}\left(z, z^{*}\right) \geq\left[\left\langle\widetilde{a_{n}}, z^{*}\right\rangle+\left\langle z-\widetilde{a_{n}}, \widetilde{a}_{n}^{*}\right\rangle+\left\langle z-\tilde{a}_{n}, b_{n}^{*}\right\rangle\right] \\
\Rightarrow & F_{A+B}\left(z, z^{*}\right) \geq\left\langle\widetilde{a_{n}}, z^{*}\right\rangle-K_{0}\left\|a_{n}^{*}\right\| n+\left\langle z-\widetilde{a_{n}}, b_{n}^{*}\right\rangle \\
\Rightarrow & \frac{F_{A+B}\left(z, z^{*}\right)}{\left\|b_{n}^{*}\right\|} \geq\left\langle\tilde{a_{n}}, \frac{z^{*}}{\left\|b_{n}^{*}\right\|}\right\rangle-\frac{K_{0}\left\|a_{n}^{*}\right\|}{\left\|b_{n}^{*}\right\|}+\left\langle z-\widetilde{a_{n}}, \frac{b_{n}^{*}}{\left\|b_{n}^{*}\right\|}\right\rangle
\end{aligned}
$$

By Banach-Alaoglu Theorem [11, Theorem 3.15], there exist a weak $k^{*}$ convergent subnet $\left(\frac{b_{\gamma}^{*}}{\left\|b_{\gamma}^{*}\right\|}\right)$ of $\left(\frac{b_{n}^{*}}{\left\|b_{n}^{*}\right\|}\right)$ such that

$$
\frac{b_{\gamma}^{*}}{\left\|b_{\gamma}^{*}\right\|} \longrightarrow v_{\infty}^{*} \in X^{*}
$$

Using (17) and taking limit in (16) along the subnet, we have $\left\langle z-\beta z, v_{\infty}^{*}\right\rangle \leq 0$

$$
\left\langle z, v_{\infty}^{*}\right\rangle \leq 0
$$

On the other hand, since $0 \in \operatorname{int} \operatorname{dom} B$ by using Fact 2 , there exist $\varepsilon>0$ and $M>0$ such that

$$
\sup _{y^{*} \in B y}\left\|y^{*}\right\| \leq M, \quad \forall y \in \varepsilon \mathbb{U}_{\mathbb{X}}
$$


Since $\left(\widetilde{a_{n}}, b_{n}^{*}\right) \in \operatorname{gra} B$, then we have

$$
\begin{aligned}
& \left\langle\widetilde{a_{n}}-y, b_{n}^{*}-y^{*}\right\rangle \geq 0, \quad \forall y \in \varepsilon \mathbb{U}_{\mathbb{X}}, y^{*} \in B(y), n \in \mathbb{N} \\
\Rightarrow & \left\langle\widetilde{a_{n}}, b_{n}^{*}\right\rangle-\left\langle y, b_{n}^{*}\right\rangle+\left\langle\widetilde{a_{n}}-y,-y^{*}\right\rangle \geq 0 \quad \forall y \in \varepsilon \mathbb{U}_{\mathbb{X}}, y^{*} \in B(y), n \in \mathbb{N} \\
\Rightarrow & \left\langle\widetilde{a_{n}}, b_{n}^{*}\right\rangle-\left\langle y, b_{n}^{*}\right\rangle \geq-\left(\left\|\widetilde{a_{n}}\right\|+\varepsilon\right) M, \quad \forall y \in \varepsilon \mathbb{U}_{\mathbb{X}}, n \in \mathbb{N} \\
\Rightarrow & \left\langle\widetilde{a_{n}}, b_{n}^{*}\right\rangle \geq \varepsilon\left\|b_{n}^{*}\right\|-\left(\left\|\widetilde{a_{n}}\right\|+\varepsilon\right) M, \quad \forall n \in \mathbb{N} \\
\Rightarrow & \left\langle\widetilde{a_{n}}, \frac{b_{n}^{*}}{\left\|b_{n}^{*}\right\|}\right\rangle \geq \varepsilon-\frac{\left(\left\|\widetilde{a_{n}}\right\|+\varepsilon\right) M}{\left\|b_{n}^{*}\right\|}, \quad \forall n \in \mathbb{N} .
\end{aligned}
$$

Using (17) and taking limit in (20) along the subnet, we have $\left\langle\beta z, v_{\infty}^{*}\right\rangle \geq \varepsilon>0$ which contradict to (18). Hence $F_{A+B}\left(z, z^{*}\right) \geq\left\langle z, z^{*}\right\rangle$.

Proposition 1. Let $A: X \rightrightarrows X^{*}$ be of type (FPV), and let $B: X \rightrightarrows X^{*}$ be maximally monotone. Let $\left(z, z^{*}\right) \in X \times X^{*}$, $x_{0} \in \operatorname{dom} A \cap \operatorname{dom} B$ and for every $x \in \operatorname{dom} A \cap$ int $\operatorname{dom} B,\left\|x^{*}\right\| \leq|B(x)|, x^{*} \in A(x)$ holds. Assume that there exists $\left(a_{n}\right)_{n \in \mathbb{N}} \in$ $\overline{\operatorname{dom} A} \cap$ bdry $\overline{\operatorname{dom} B}$ such that it converges to a point in $\left[x_{0}, z\right]$. Then $F_{A+B}\left(z, z^{*}\right) \geq\left\langle z, z^{*}\right\rangle$.

Proof.Assume to the contrary

$$
F_{A+B}\left(z, z^{*}\right)<\left\langle z, z^{*}\right\rangle \text {. }
$$

By the necessary translation if necessary, we can suppose that $x_{0}=0 \in \operatorname{dom} A \cap \operatorname{int} \operatorname{dom} B$ and $(0,0) \in \operatorname{gra} A \cap \operatorname{gra} B$. By the assumption that, there exists $0 \leq \beta<1$ such that

$$
a_{n} \longrightarrow \beta z
$$

Since $0 \in \operatorname{int} \operatorname{dom} B$ and by (21) Fact 2, we have

$$
0<\beta<1 \text { and } \beta z \neq 0
$$

By the similar argument of Lemma 2, there exists $\left(\widetilde{a_{n}},{\widetilde{a_{n}}}^{*}\right)_{n \in \mathbb{N}}$ in $\operatorname{gra} A \cap\left(H_{n} \times X^{*}\right)$ such that

$$
\left\langle z-\widetilde{a_{n}},{\widetilde{a_{n}}}^{*}\right\rangle \geq-K_{0}\left\|a_{n}^{*}\right\|
$$

where $K_{0}=\frac{1}{\beta^{2}}(2\|z\|+2)$. Since $\beta z \in$ bdry $\operatorname{dom} B$ we consider two cases:

Case 1. $\beta z \notin \operatorname{dom} B$. By the same argument of Lemma 2, we obtain a contradiction.

Case 2. $\beta z \in \operatorname{dom} B$. Since $\beta z \in$ bdry $\operatorname{dom} B$. Take $y_{0}^{*} \in N_{\overline{\operatorname{dom} B}}(\beta z)$ such that

$$
\left\langle y_{0}^{*}, \beta z-y\right\rangle>0, \text { for every } y \in \text { int } \operatorname{dom} B .
$$

Thus, $t y_{0}^{*} \in N \overline{\overline{\operatorname{dom} B}}(\beta z), \forall t>0$. Since $\beta z \in \overline{\operatorname{dom} \mathrm{A}}$, we again consider the following two subcases:

Subcase 1. $\beta z \in \operatorname{dom} A$. Since $0 \in$ int $\operatorname{dom} B$ then by (25), we have

$$
\left\langle y_{0}^{*}, z\right\rangle>0 .
$$

Since $B$ is maximally monotone. By [13, Lemma 28.5], $B=B+N \overline{\operatorname{dom} B}$ and $\beta z \in \operatorname{dom} A \cap \operatorname{dom} B$. Then we compute

$$
F_{A+B}\left(z, z^{*}\right) \geq \sup \left[\langle z-\beta z, A(\beta z)\rangle+\left\langle z-\beta z, B(\beta z)+t y_{0}^{*}\right\rangle+\left\langle z^{*}, \beta z\right\rangle\right] .
$$


Thus,

$$
\frac{F_{A+B}\left(z, z^{*}\right)}{t} \geq \sup \left[\left\langle z-\beta z, \frac{A(\beta z)}{t}\right\rangle+\left\langle z-\beta z, \frac{B(\beta z)}{t}+y_{0}^{*}\right\rangle+\frac{\left\langle z^{*}, \beta z\right\rangle}{t}\right] .
$$

By (21), letting $t \rightarrow \infty$ we have $\left\langle z-\beta z, y_{0}^{*}\right\rangle \leq 0$ and since $\beta<1$ we obtain

$$
\left\langle z, y_{0}^{*}\right\rangle \leq 0
$$

which contradicts to (26).

Subcase 2. $\beta z \notin \operatorname{dom} A$. Set $U_{n}:=\beta z+\frac{1}{n} \mathbb{U}_{\mathbb{X}}$. Since $\beta z \in \overline{\operatorname{dom}} A$ we have $\operatorname{dom} A \cap U_{n} \neq \phi$. Since $\left(\beta z, \beta z^{*}\right) \notin \operatorname{gra} A$. By using (FPV) property of $A$, there exists $\left(\widetilde{a_{n}}, \widetilde{a}_{n}{ }^{*}\right) \in \operatorname{gra} A \cap\left(U_{n} \times X^{*}\right)$ such that

$$
\left\langle\beta z-\widetilde{a}_{n}, \beta z^{*}-{\widetilde{a_{n}}}^{*}\right\rangle<0
$$

which implies that

$$
\begin{aligned}
& \left\langle\beta z-\widetilde{a_{n}},{\widetilde{a_{n}}}^{*}\right\rangle>\left\langle\beta z-\widetilde{a_{n}}, \beta z^{*}\right\rangle \\
\Rightarrow & \left\langle z-\widetilde{a_{n}},{\widetilde{a_{n}}}^{*}\right\rangle-\frac{(1-\beta)}{\beta}\left\langle\widetilde{a_{n}},{\widetilde{a_{n}}}^{*}\right\rangle>\left\langle\beta z-\widetilde{a_{n}}, \beta z^{*}\right\rangle \\
\Rightarrow & \left\langle z-\widetilde{a_{n}},{\widetilde{a_{n}}}^{*}\right\rangle>\frac{(1-\beta)}{\beta}\left\langle\widetilde{a_{n}},{\widetilde{a_{n}}}^{*}\right\rangle+\left\langle\beta z-\widetilde{a_{n}}, \beta z^{*}\right\rangle .
\end{aligned}
$$

Since $(0,0) \in \operatorname{gra} A$ and $\left(\widetilde{a_{n}},{\widetilde{a_{n}}}^{*}\right) \in \operatorname{gra} A$. By monotonicity of $A,\left\langle\widetilde{a_{n}},{\widetilde{a_{n}}}^{*}\right\rangle \geq 0$. Appealing to the above equation, we have

$$
\left\langle z-\widetilde{a_{n}},{\widetilde{a_{n}}}^{*}\right\rangle \geq\left\langle\beta z-\tilde{a_{n}}, z^{*}\right\rangle
$$

Since $\beta z \in$ bdry $\operatorname{dom} B$. By $0 \in$ int $\operatorname{dom} B$, Fact 2 and Lemma 1 , we have $\beta z \in \operatorname{int}(1+\varepsilon) \operatorname{dom} B$, for every $0<\varepsilon<1$. Since $\widetilde{a_{n}} \rightarrow \beta z$. Thence, there exists $n_{0} \in \mathbb{N}$ such that $\tilde{a_{n}} \in \operatorname{int}(1+\varepsilon) \operatorname{dom} B, \forall n \geq n_{0}$. Thus, for every $0<\varepsilon<1, \widetilde{a_{n}} \in \operatorname{int}(1+$ $\varepsilon) \operatorname{dom} B, \forall n \geq n_{0}$. Therefore, $\widetilde{a_{n}} \in \overline{\operatorname{dom} B} \forall n \geq n_{0}$. By Fact 2, we have $\widetilde{a_{n}} \in \operatorname{dom} B$. Since $\widetilde{a_{n}} \in \operatorname{dom} A$ and $\widetilde{a_{n}} \rightarrow \beta z$, then by Fact 2 , we have $\widetilde{a_{n}} \in$ int $\operatorname{domB}$. Thus, $\widetilde{a_{n}} \in \operatorname{dom} A \cap$ int domB and hence by hypothesis, there exists some $b_{n}^{*} \in B\left(\widetilde{a_{n}}\right)$, such that $\left\|{\tilde{a_{n}}}^{*}\right\| \leq\left\|b_{n}^{*}\right\|$. By Fact 2, $\left\|{\widetilde{a_{n}}}^{*}\right\| \rightarrow+\infty$. Hence $\left\|b_{n}^{*}\right\| \geq n\left\|a_{n}^{*}\right\|$ for all $n \in \mathbb{N}$ and $a_{n}^{*} \in X^{*}$ That is (14) of Lemma 2 holds. Then by the same argue of Lemma 2 we obtain a contradiction. Hence $F_{A+B}\left(z, z^{*}\right) \geq\left\langle z, z^{*}\right\rangle$.

Proposition 2. Let $A: X \rightrightarrows X^{*}$ be of type (FPV), and let $B: X \rightrightarrows X^{*}$ be maximally monotone with (i) $\operatorname{dom} B$ is open or (ii) for every $x \in$ dom $A \cap$ int dom $B,\left\|x^{*}\right\| \leq|B(x)|, x^{*} \in A(x)$ holds and $\operatorname{dom} A \cap$ int $\operatorname{dom} B \neq \phi$. Suppose that there exists $\left(z, z^{*}\right) \in X \times X^{*}$ such that $F_{A+B}\left(z, z^{*}\right)<\left\langle z, z^{*}\right\rangle$. Then $z \in \overline{\operatorname{dom} A}$.

Proof. By the necessary translation if necessary, we can suppose that $0 \in \operatorname{dom} A \cap$ int $\operatorname{dom} B$ and $(0,0) \in \operatorname{gra} A \cap \operatorname{gra} B$. We assume to the contrary that

$$
z \notin \overline{\operatorname{dom} A} \text {. }
$$

By using equation (28) and Fact 2 , we have there exist $\left(a_{n}, a_{n}^{*}\right)_{n \in \mathbb{N}}$ in $\operatorname{gra} A$ and $0 \leq \lambda<1$ such that

$$
\left\langle z-a_{n}, a_{n}^{*}\right\rangle \longrightarrow+\infty \text { and } a_{n} \longrightarrow \lambda z
$$

Now we consider the following cases.

Case 1. There exists a subsequence of $\left(a_{n}\right)_{n \in \mathbb{N}}$ in $\operatorname{dom} B$.

We can suppose that $a_{n} \in \operatorname{dom} B$ for every $n \in \mathbb{N}$. Thus by 29 and Fact 2 , we have $F_{A+B}\left(z, z^{*}\right)=+\infty$, which is a 
contradiction to the hypothesis that $F_{A+B}\left(z, z^{*}\right)<\left\langle z, z^{*}\right\rangle$.

Case 2. There exists $n_{1} \in \mathbb{N}$ such that $a_{n} \notin \operatorname{dom} B$ for every $n \geq n_{1}$.

Now we suppose that $a_{n} \notin \operatorname{dom} B$ for every $n \in \mathbb{N}$. Since $a_{n} \notin \operatorname{dom} B$, by Fact 2 and Fact 2, there exists $\beta_{n} \in[0,1]$ such that

$$
\beta_{n} a_{n} \in \text { bdry } \overline{\operatorname{dom} B} \text {. }
$$

By equation (29), we can suppose that

$$
\beta_{n} a_{n} \longrightarrow \beta z
$$

Since $0 \in \operatorname{int} \operatorname{dom} B$ then by (28) and Fact 2 , we have

$$
0<\beta<1 .
$$

If (i) holds then by Lemma 2, we have $F_{A+B}\left(z, z^{*}\right) \geq\left\langle z, z^{*}\right\rangle$ and if (ii) hold. By Proposition $1, F_{A+B}\left(z, z^{*}\right) \geq\left\langle z, z^{*}\right\rangle$ which is a contradiction. Hence by combining all the above cases, we have proved that $z \in \overline{\operatorname{dom} A}$.

Theorem 1. [Main result] Let $A, B: X \rightrightarrows X^{*}$ be maximally monotone with (i) $\operatorname{dom} B$ is open or (ii)for every $x \in$ domA $\cap$ int dom $B,\left\|x^{*}\right\| \leq|B(x)|, x^{*} \in A(x)$ holds and $\operatorname{dom} A \cap \operatorname{int} \operatorname{dom} B \neq \phi$. Assume that $A$ is of type $(F P V)$. Then $A+B$ is maximally monotone.

Proof. By the necessary translation if necessary, we can suppose that $0 \in \operatorname{dom} A \cap \operatorname{int} \operatorname{dom} B$ and $(0,0) \in \operatorname{gra} A \cap \operatorname{gra} B$. From Fact 2, we have $\operatorname{dom} A \subseteq P_{X}\left(\operatorname{dom} F_{A}\right)$ and $\operatorname{dom} B \subseteq P_{X}\left(\operatorname{dom} F_{B}\right)$. Thus,

$$
0 \in \operatorname{Core}[\operatorname{Conv}(\operatorname{dom} A)-\operatorname{Conv}(\operatorname{dom} B)]
$$

Hence

$$
\bigcup_{\lambda>0} \lambda\left(P_{X}\left(\operatorname{dom} F_{A}\right)-P_{X}\left(\operatorname{dom} F_{B}\right)\right)=X .
$$

Thus, by Fact 2 it is sufficient to prove that

$$
F_{A+B}\left(z, z^{*}\right) \geq\left\langle z, z^{*}\right\rangle, \quad \forall\left(z, z^{*}\right) \in X \times X^{*} .
$$

Let $\left(z, z^{*}\right) \in X \times X^{*}$. On the contrary assume that

$$
F_{A+B}\left(z, z^{*}\right)<\left\langle z, z^{*}\right\rangle
$$

Then by equation (34) Proposition 2 and Fact 2 we have

$$
z \in \overline{\operatorname{dom} A} \backslash \overline{\operatorname{dom} B} .
$$

Since $z \in \overline{\operatorname{dom} A}$, there exists $\left(a_{n}, a_{n}^{*}\right)_{n \in \mathbb{N}}$ in gra $A$ such that

$$
a_{n} \longrightarrow z \text {. }
$$

By (35), $a_{n} \notin \overline{\operatorname{dom} B}$ for all but finitely many terms $a_{n}$. We can suppose that $a_{n} \notin \overline{\operatorname{dom} B}$ for all $n \in \mathbb{N}$. By Fact 2 and Fact 2 , there exists $\left.\beta_{n} \in\right] 0,1[$ such that

$$
\beta_{n} a_{n} \in \text { bdry } \overline{\operatorname{dom} B} \text {. }
$$


By (36) and $\beta \in[0,1]$ we have

$$
\beta_{n} a_{n} \longrightarrow \beta z \text {. }
$$

By (37) and (35) we have $0<\beta<1$. If (i) hold, by Lemma 2, we have a contradiction and if (ii) hold, by Proposition 1, we obtain a contradiction. Thus, we have $F_{A+B}\left(z, z^{*}\right) \geq\left\langle z, z^{*}\right\rangle$ for all $\left(z, z^{*}\right) \in X \times X^{*}$. Hence $A+B$ is maximally monotone.

\section{Competing interests}

The authors declare that they have no competing interests.

\section{Authors' contributions}

All authors have contributed to all parts of the article. All authors read and approved the final manuscript.

\section{References}

[1] H. H. Bauschke, X. Wang and L. Yao, An answer to S. Simons' question on the maximal monotonicity of the sum of a maximal monotone linear operator and a normal cone operator, Set-Valued Var. Anal. 17 (2009) 195-201.

[2] H. H. Bauschke, X. Wang and L. Yao, On the maximal mono tonicity of the sum of a maximal monotone linear relation and the subdifferential operator of a sublinear function, Proceedings of the Haifa Workshop on Optimization Theory and Related Topics. Contemp. Math., Amer. Math. Soc., Providence, RI 568 (2012) 19-26.

[3] J. M. Borwein, Maximality of sums of two maximal monotone operators in general Banach space, P. Am. Math. Soc. 135 (2007) 3917-3924.

[4] J. M. Borwein and L. Yao, Maximality of the sum of a maximally monotone linear relation and a maximally monotone operator, Set-Valued Var Anal. 21 (2013) 603-616.

[5] J. M. Borwein and L. Yao, Structure theory for maximally monotone operators with points of continuity, J. Optim Theory Appl. 157 (2013) 1-24 http://dx.doi.org/10.1007/s10957-012-0162-y.

[6] J.M. Borwein and L. Yao, Sum theorems for maximally monotone operators of type (FPV), J. Aust. Math. Soc. 97 (2014) 1-26.

[7] S. Fitzpatrick, Representing monotone operators by convex functions, in Work- shop/Miniconference on Functional Analysis and Optimization (Canberra 1988), Proceedings of the Centre for Mathematical Analysis, Australian National University, Canberra, Australia, 20 (1988) 59-65.

[8] R.R. Phelps, Convex Functions, Monotone Operators and Differentiability, 2nd Edition, Springer-Verlag, 1993.

[9] R.T. Rockafellar, Local boundedness of nonlinear, monotone operators, Mich. Math. J. 16 (1969) 397-407.

[10] R.T. Rockafellar, On the maximality of sums of nonlinear monotone operators, T. Am. Math. Soc. 149 (1970) 75-88.

[11] R. Rudin, Functional Analysis, Second Edition, McGraw-Hill, 1991.

[12] S. Simons, Minimax and Monotonicity, Springer-Verlag, 1998.

[13] S. Simons, From Hahn-Banach to Monotonicity, Springer-Verlag, 2008.

[14] M.D. Voisei, The sum and chain rules for maximal monotone operators, Set-Valued Var. Anal. 16 (2008) 461-476.

[15] L. Yao, The sum of a maximally monotone linear relation and the subdifferential of a proper lower semicontinuous convex function is maximally monotone, Set-Valued Var. Anal. 20 (2012) 155-167.

[16] L. Yao, Maximality of the sum of the subdifferential operator and a maximally monotone operator, arXiv: 1406.7664v1[math.FA] 30 Jun 2014, http://arxiv.org/pdf/1406.7664.pdf.

[17] C. Zalinescu, Convex Analysis in General Vector Spaces, World Scientific Publishing, 2002. 